\title{
The Teaching of the Concept of Color Harmony and Its Ambiguity
}

\author{
Ng Woon Lam \\ School of Art, Design \& Media, Nanyang Technological University, Singapore \\ Received: April 10, $2021 \quad$ Accepted: May 6, $2021 \quad$ Published: August 30, 2021
}

To cite this article: Ng Woon Lam. (2021). The teaching of the concept of color harmony and its ambiguity, Asia-Pacific Journal of Humanities and Social Sciences, 01:2, 020-027, DOI: 10.53789/ j.1653-0465.2021.0102.002

To link to this article: https://doi.org/10.53789/j.1653-0465.2021.0102.002

\begin{abstract}
This paper aims to address a long-term issue in the teaching of practical color theory knowledge - the concept of color harmony. The concept of color harmony has been used as the pedagogical framework for students' learning of color application. On the contrary, multiple quantitative studies, which are cited in the paper, have shown evidence that the ambiguity of this concept could not be addressed. The research process started by looking into how the concept of color harmony was derived. It was then compared with the practical method, the Munsell Color System, which was developed to fulfil industrial needs. Multiple quantitative research studies have shown the ambiguity of the concept of color harmony. Therefore, this paper suggests that for the development of a pedagogical framework of color theory, the concept of color harmony should not be included. Instead, the Munsell Color System, which is a closer practical model, should be adopted so that the learning connects to a true and practical difference between colors instead of an ambiguous philosophical concept of harmony.
\end{abstract}

Keywords: Munsell; color; harmony

Notes on the contributor: Dr. Ng Woon Lam is currently an associate professor at the School of Art, Design \& Media (ADM), Nanyang Technological University (NTU), Singapore. He teaches Water Media Landscape Painting and Foundation Drawing at ADM. His research areas include color theory, Chinese calligraphy, Chinese and Western paintings.

\section{Background}

The practical concept of color harmony has a strong connection with the development of Newton's closedring model of color placement based on Newton's Optics ( Newton 1704).

Scientist Isaac Newton discovered the spectrum of light through the prism-dissected process (Martins, R. and Silva, C. 2001). It was only understood later in the late nineteenth century with the scientific advancement in pigment manufacturing and light optics that the mixing of light is an additive process, which is the opposite of the mixing of pigments. The latter is subtractive (Shapiro, A 1994). However, that confusion did not affect its influence on the development of color theory. Newton's Optics has since influenced the study of color theory in two ways, namely the dissection of the spectral hues of sunlight and his closed-ring arrangement of chromatic 


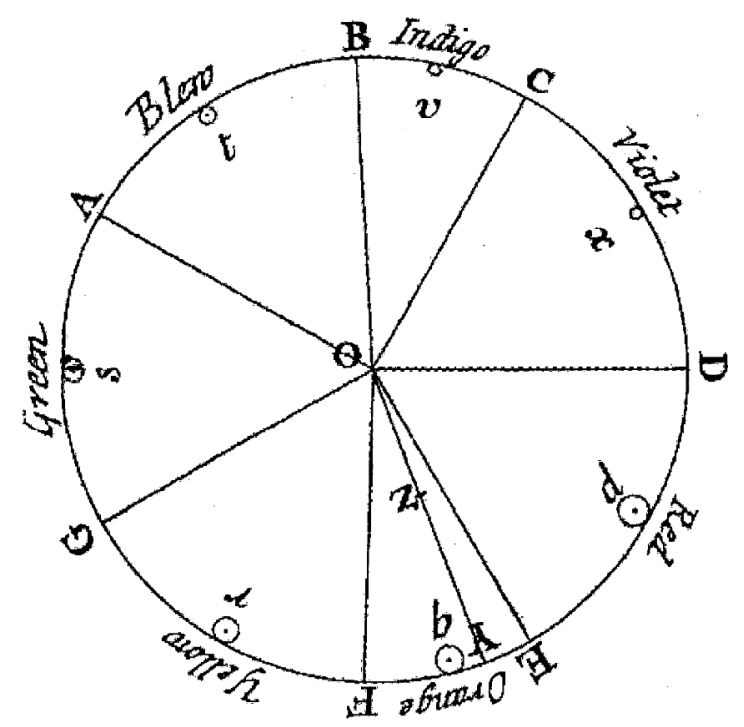

Figure 1 Newton's Color Circle based on Newton's Optics

hues, as shown in Figure 1. He added a purple hue to closed the gap by bridging red and violet in his spectral layout. The current popular color theory learning tool, the symmetrical color wheel also rooted in Newton's close -ring model, which was developed by Johannes Itten, a professor at the Bauhaus during the early 20th century (Itten, J. 1962). Figure 2 is the popular color wheel that is available at most art stores. It adopts the Itten Color System.

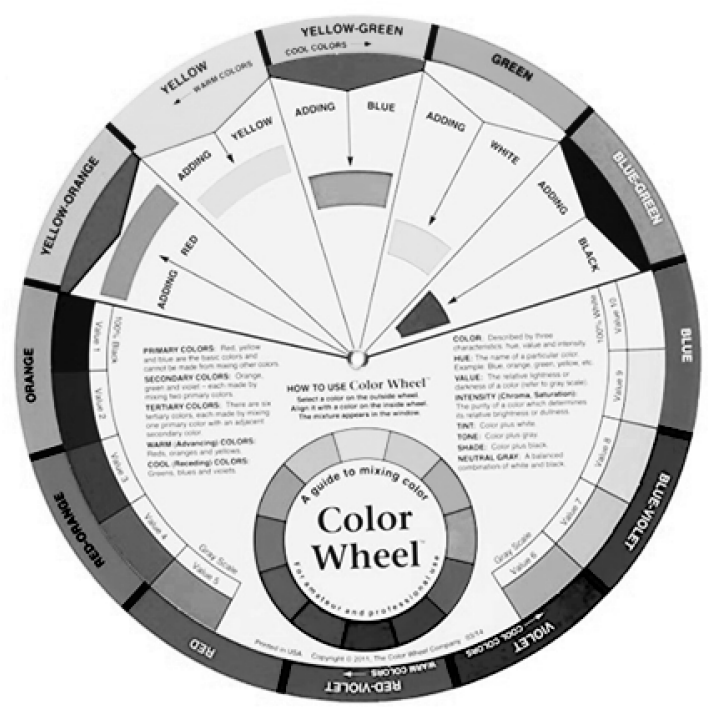

Figure 2 A commercially available color wheel based on the Itten Color System

This arrangement clearly indicates the progressive difference between chromatic hues, although Newton arbitrarily selected six hues from the continuous visible spectrum dissected while added a purple hue to connect the two hues-red and violet hues at the two ends of the spectrum. Other non-spectral colors, the browns, and grays were omitted in his model. In some of the recent commercial reference models, the intermediate colors, the browns and grays have been added to depict the practical relationship of the chromatic hues and these intermediate colors. However, the symmetrical Itten model was not first developed with this practical consideration in mind. It 
originated from the Greek living philosophy. The concept of color harmony was discussed in Burchett's publication (Burchett, K. E. 2005)

The concept of living in harmony may carry distinctive meanings based on different cultural contexts, as studied by Yu. "Harmony" in the Chinese culture is based on the Confucian living philosophy, while the Greeks regard it as a perfect order that pre-exists on earth ( YU, J. 2016). The latter was used to construct the concept of color harmony. The concept of color harmony used for the development of practical applications of colors could be categorized into two stages, namely from the Renaissance to before Impressionism and from Impressionism to Modern (Lyon, C. 1991). The shift was connected with the scientific advancement of chemistry and manufacturing technology. Prismatic pigments were only available years after the Industrial Revolution when factories could manufacture tube pigments in bulk with new chemical knowledge (Lyon, C. 1991). Therefore, the transitional period happened during the early nineteenth century - the period of Romanticism right before Impressionism.

During the Renaissance, prismatic pigments were limited. Most of them, like indigo blue, red madder or ultramarine, were from naturally formed crystals or plants and animals. The impurities in these pigments were higher (Price, M. 2000). Therefore, It was harder for the Renaissance painters to achieve high chroma as compared to factory-manufactured prismatic pigments used by the Impressionists. In addition, new chemical knowledge and manufacturing technology advancement also provided a wider range of prismatic colors for the Impressionists (Lyon, C. 1991).

Using Leonardo Da Vinci's paintings as examples, during the Renaissance period, the most abundant pigments other than the rare and prismatic crystals mentioned above were mostly earth materials. Therefore, the most common colors were less chromatic browns and grays. Leonardo used his chiaroscuro painting concept that depended more on tone to model forms. That was regarded as the first type of color harmony. The artist kept the hue character of an object while modeling the forms with tonal range to achieve a sense of three dimensions (Shearman, J. 1962). Leonardo constructs space and forms through a wide range of tones while limiting large hue shifts - the use of light and shadows.

Renaissance painters like Andrea del Sarto, Rosso, and Pontormo applied the same concept to depict forms and space. The hue of the shadow areas of an object would be similar to the lighted areas. It creates a believable transition and presents a three-dimensional visual form. This is regarded as color harmony since forms were presented convincingly in space ( $\mathrm{Ng}$, W. L. 2018). It was later named analogous color harmony when different types of color harmony were introduced.

When Newton introduced the layout of his closed-ring model of prismatic hues (Newton 1704), comparing hue differences became equally important besides the above-mentioned tonal unity. Combining the discovery of the simultaneous contrast of colors by a chemist, Chevreul, in 1839, a new form of color harmony started to develop and later flourished during the Impressionist period (Roque, G. 1996). In practical terms, simultaneous contrast of colors means the hues of any two different colors will create a dynamic influence on each other when they are placed adjacently. Both hues shift away from each other and show larger hue contrast. Artists started to use the closed-ring color arrangement - the color wheel as a guide to decide color combinations (Martins, R. and Silva, C. 2001), and later a new form of color harmony, the Contrast Harmony was introduced by pairing complementary hues on the opposite sides of the ring to create hue contrast. 


\section{Discussions}

The Munsell Color Space developed by Professor Albert Munsell (1902) was constructed to include three attributes of colors, namely value, hue, and chroma, forming a three-dimensional color space. He extended the effort to mathematically describe the two forms of color harmony described above ${ }^{1}$. The mathematical concept can be summarized as five points below:

- Grays, which also include black and white, are centralized as the core of the Munsell Color Space, assumed to be neutral and are therefore in perfect harmony.

- Colors with different values (lightness) but with similar hues and chroma are considered in harmony.

- Colors on the opposite ends, working as complementary colors which have the same values are considered harmonic pairs.

- One color may be combined with another. The chromaticity and hue of this color will decrease according to the Munsell Color Space. These colors are considered harmonious.

- Colors selected along the elliptical locus in the Munsell Color Space are considered in harmony.

The first two concepts were derived from the concept of tonal unity used during the Renaissance before Impressionism. The third concept was the Contrast Harmony concept, while the last two were an expansion of the concept of color harmony to include more colors than a sole grouping of similar hue colors or pairing of only two complementary colors.

Simultaneous contrast is also evidence of the dynamic nature of human vision. Any physical measurement of color may obtain a precise value. However, due to the dynamics of human vision, the viewer's response is not constant. Furthermore, the argument that suggests neutrals grays are in a state of harmony has no scientific proof ( Ng, W. L. 2018). The cultural difference may further complicate the issue.

Koenig categorized color harmony into three types (Westland, S. 2007):

- Simple harmony: Colors with a similar or small shift in hue with a different tone and chroma. The major contrast is caused by the tonal difference in an application as the hue range is limited. It is also called analogous colors in practice today.

- Contrast harmony: Using the law of simultaneous contrast, colors with a vastly different hue may be paired or grouped and used. In an application, the contrast may come from hue, tone, and chroma. The maximum difference between these opposite colors can be seen as a large vector formed in a color space ( $\mathrm{Ng}$, W. L. 2018). In practice, the color pairs are named complementary colors.

- Balanced harmony: It is an extension of the contrast harmony to include practical situations.

Complementary colors also have multiple definitions:

- Two chromatic pigments that mixed to obtain a neutral gray.

- Two chromatic hues thatare painted as sectors on a wheel that spins to form a neutral gray. 
Defined by Mahyar and peers, “... complementary colors shall be defined as those colors which would spin to gray on a color wheel.” (Judd, D. B. 1963 ).

- A chromatic color image is seen for a short period (between 10 to 20 seconds based on my teaching experience), an afterimage is formed in the viewer's mind. The presented hue and the afterimage hue in the viewer's mind form a complementary pair ( Westland, S. 2007).

Measurements carried out could not align well with definitions (Mahyar, F. 2007). Viewers did not select the same pairings when the questions were reversed. The dynamic nature of visual response might further influence the consistency of the measurements. The ambiguity of the harmony is even more evident when one needs to know the proportion of how much each hue is to be applied under a practical situation ( $\mathrm{Ng}$, W. $\mathrm{L}$. 2018 ). No guideline can be given for the right proportion of each hue to be applied.

The initial concept of color harmony was derived from the Greek philosophy of musical harmonic intervals (Kuehni, R.G. 1990). Newton also arbitrarily selected seven colors for this closed-ring color arrangement while the actual spectrum of light is continuous. He added a purple color to complete his color circle - the closed-ring model (Gage, J. 1999). This has influenced the research, teaching, and learning of color theory for more than 300 years.

Color harmony was also linked to how the color pairing or grouping may generate a pleasing effect (Judd, D. B. 1963). Granville and Kuehni have proven otherwise through contextual differences between cultures, indicating that color harmony based on a "pleasing” outcome cannot be well quantified ( Collins, J. 2004). Westland further argued that color choice could change according to race, age, cultural background, education, time, and other factors which introduce more unpredictable variables (Westland, S. 2007). Therefore, the quantification of color harmony is almost impossible.

$\mathrm{Ou}$ also investigated the concept of color harmony based on the feeling of pleasantness and the order of colors ( OU, L. C. and LUO, M. R. 2006). According to Ou, since pleasantness was connected to emotional response, a measurement of a person's emotional state could be equivalent. He also added the measurement of aesthetics using terms by other color scientists, namely, complementary (Chevreul, M. E. 1981), order (Ostwald, W. 1969), balance (Munsell, A. 1969), and unambiguity (Moon, P. and Spencer, D. 1944). All these parameters were related to the concept of color harmony. These parameters were even more difficult to quantify. In addition, the other two attributes of colors, tone, and chroma could further increase the complexity in the quantification process. Hence, he concluded that color harmony could happen in any combination ( $\mathrm{Ng}$, W. L. 2018).

Ou concluded that "In the present study, we have assumed that regardless of which color selection method is applied, a harmonious combination can always give viewers a pleasing impression.”-- ( OU, L. C. and LUO, M. R. 2006).

$\mathrm{Ou}$ also measured the order of colors and confirmed that color order could not always construct harmony ( OU, L. C. and LUO, M. R. 2006). I would argue that this concept of color harmony is an overly idealistic concept that does not exist in the first place.

Besides the pairing concept of colors, there is another important practical issue that the concept of color harmony has induced to the learning of color theory. The concept was first introduced through a symmetrical color wheel model derived from Newton's closed-ring color arrangement (Newton 1704). The idea of each hue 

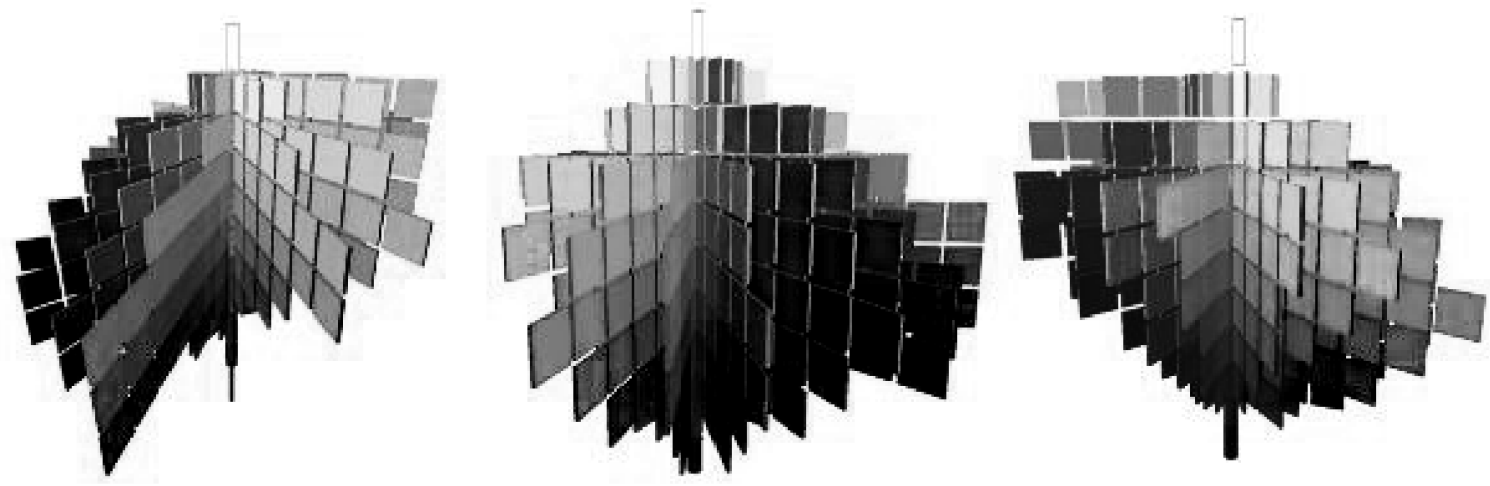

Figure 3 Three different angles of the Munsell Color Space, using RGB conversion ( Ng, W. L. 2018)

having a similar practical range somehow is ironed into many beginners' minds through their immediate acceptance of a symmetrical color wheel. The most popular one is the Itten Color Wheel (Itten, J. 1962) that is available in many art stores, as shown in Figure 2. Professor Munsell, during his development of the Munsell Color Space (1902), has practically addressed that each hue has a very different working range, as shown in Figure 3. For example, when a yellow hue to shaded, as shown in Figure 4, the yellow hue shift towards the green hue region. This was also discussed by Kopacz in her publication - "Color in Three-Dimensional Design" (Kopacz, J. 2003) and she also referred to the Munsell Color Space.

Kopacz mentioned that "However, yellow has very few steps in shade range because it turns greenish as it darkens.” (Kopacz, J. 2003).

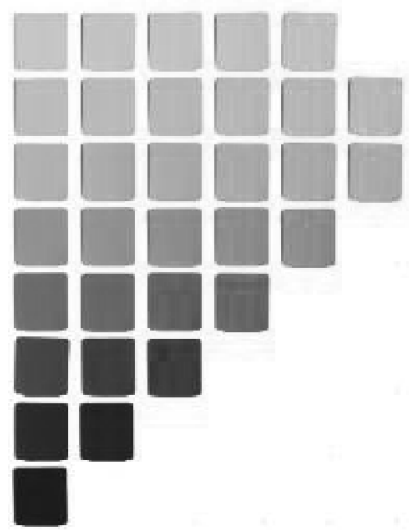

Figure 4 Hue 7.5 Y of the Munsell Color Book indicates that the yellow hue shifts towards the green hue when it is shaded

Some complementary color pairings will therefore be affected by this shift. When a complementary color pair is a yellow and a purple hue, the shaded yellow is practically a green hue. Therefore, that is effectively a threehue system instead of two. If a user does not understand the asymmetrical nature of the practical color space, this issue is not well addressed ( Ng, W. L. 2015). A similar situation will happen to red and orange hues that will shift towards purple and dark brown, respectively, when they are shaded.

From 2005 to 2019, a series of efforts were carried out by the Budapest University of Technology and Economics to measure color harmony. There was hardly any approach that could help educators construct a reliable pedagogy for the training of color harmony. However, the color harmony concept is still adopted as a color teaching guide today. "Design Elements - Color Fundamentals" by Sherin (Sherin, A. 2012) and 
"Understanding Color - An Introduction for Designers" by Holtzschue (Holtzschue, L. 2006) are examples in the design area. Color harmony is discussed comprehensively in Holtzschue's publication (Holtzschue, L. 2006).

\section{Conclusion and Recommendation}

From the background information and the discussions, it is evident that the concept of color harmony could hardly form a structured pedagogy for constructing a sound color theory training. Effective teaching and learning of practical color theory and applications should assist students in recognizing the true practical range and differences between colors. On the contrary, the concept of color harmony tries to impose to beginners predefined pairing or grouping models with minimum practical consideration. When these models become color formulae in these beginners' minds, it is harder for them to comprehend the true practical differences between hues.

On the contrary, an asymmetrical color space, using the Munsell Color Space as an example, allows students to see the practical connections between colors. The transition is well represented through the connections of brown and grays with all the chromatic hues. A student does not have to memorize any pairing of colors because they are able to physically map the difference between any two colors, including chromatic and achromatic colors ( Ng, W. L. 2018). The mapping idea depends solely on the vector difference between any two colors, and that is the real practical outcome that an end-user needs, not the pairing of colors. In an image presentation, the artist needs to judge the practical difference of any two or more colors used together, not any kind of pairing or grouping philosophy. Students will also benefit from learning the practical complexity and usefulness of transitional colors, the browns, grays, purples, and black when transitional colors are discussed in relation to their chromatic parent hues ( Ng, W. L. 2018).

Hence the concept of color harmony should be avoided as a teaching framework for color theory. Instead, the focus should move to practical differences of colors in any application. Both Chevreul's law of simultaneous contrast and the tonal unity concept by the Renaissance painters are good models for students to learn the practical differences in color applications (Ng, W. L. 2018), but there is no need to connect them to any philosophical argument.

Note:

1 The mathematical model of the harmony concepts developed by Professor Albert Munsell can be obtained from this PhD dissertation: "A study of the teaching of color theory for visual art students at tertiary institutions in Singapore".

\section{References}

Burchett, K. E. (2005). A bibliographical history of the study and use of color from Aristotle to Kandinsky. New York: The Edwin Mellen Press, Ltd.

Collins, J. (2004). Turner, Whistler, Monet. Toronto, Paris and London, in The Burlington Magazine. London: Burlington Magazine Publications.

Chevreul, M. E. (1981). The principles of harmony and contrast of colors. New York: Van Nostrand Reinhold.

Gage, J. (1999). Color and meaning: Art, science, and symbolism. California: University of California Press.

Holtzschue, L. (2006). Understanding color: An introduction for designers. New Jersey: John Wiley \& Sons.

Itten, J. ( 1962). The art of color: The subjective experience and objective rationale of color. New York: Reinhold Publishing

Corporation. 
Judd, D. B. ( 1963). Wyszecki, G. \& Wintringham, W. color in business, science, and industry. Physics Today, $16(12)$, 74.

Kuehni, R. G. (1990). Industrial color difference: Progress and problems. Color Research \& Application, 15(5) , 261-265.

Kopacz, J. (2003). Color in three-dimensional design. New York: McGraw-Hill.

Lyon, C. (1991). Unveiling Monet.MoMa, 7, 14-23.

Martins, R. and Silva, C. (2001). Newton and colour: The complex interplay of theory and experiment.Science \& Education, 10(3), 287-305.

Mahyar, F. (2007). Investigation of complementary colour harmony in CIELAB colour space. Color Science for Industry. Hangzhou: International Color Association.

Munsell, A. (1969). A grammar of color. New York: Van Nostrand Reinhold.

Moon, P. and Spencer, D. (1944). Geometric formulation of classical color harmony, 34(1) , 46-59.

Newton, I. (1704). Opticks: A treatise of the reflections, refractions, inflections \& colours of light. London: S. Smith and B. Walford.

Nemcsics, A. and Takacs, J. (2019). Preference and harmony of neutral colours in 50-year apart. Color Research \& Application, 44 (1) , 98-105.

Nemcsics, A. (2008). Experimental determination of laws of color harmony. Color Research \& Application, 33(4) , $262-270$.

Nemcsics, A. (2011). Experimental determination of laws of color harmony. Color Research \& Application, 36(2), $127-139$.

$\mathrm{Ng}$, W. L. (2018). Comparison of practical application of browns and grays based on a vector concept: The practical strength the asymmetrical Munsell color space. Color Research \& Application, 43(6) , 840-849.

Ng, W. L. (2015). A new approach for the teaching of practical color theory. Bali: Indonesia: ITS Press.

Neumann, L., Nemcsics, A. and Neumann, A. (2005). Computational color harmony based on coloured system. Paris: The Eurographics Association.

OU, L. C. and LUO, M. R. (2006). A colour harmony model for two-colour combinations. Color Research \& Application, 31 (3) , 191 -204 .

Ostwald, W. (1969). The color primer: A basic treatise on the color system of Wilhelm Ostwald. New York: Van Nostrand Reinhold Co.

Price, M. (2000). A renaissance of color: Particle separation and preparation of Azurite for use in oil painting. The MIT Press, 33(4), 281-288

Roque, G. (1996). Chevreul and impressionism: A reappraisal. The Art Bulletin, 78(1), 26-39.

Sherin, A. (2012). Design elements: Color fundamentals. United States: Rockport Publishers.

Shapiro, A. (1994). Artists' colors and Newton's colors. The University of Chicago Press on behalf of The History of Science Society, 85 (4), 600-630.

Shearman, J. (1962). Leonardo's colour and chiaroscuro. Zeitschrift für Kunstgeschichte, 25(1) , 13-47.

The-Color-Wheel-Company.Cox Color Wheel. 2000 - 2018 [ cited 202111 May 2021]; Available from: https://colorwheelco.com.

Westland, S. (2007). Color harmony. Colour Journal: Design and Creativity, 1(1), 1-15.

YU, J. ( 2016 ). Chinese harmony and greek harmony: On Li Chenyang's the Confucian philosophy of harmony. A Journal of Comparative Philosophy, 15(3), 413.

(Editor : Jasmine) 